\title{
Nonlinear plasmonic directional couplers
}

\author{
José R. Salgueiro, ${ }^{1,2, a)}$ and Yuri S. Kivshar ${ }^{2}$ \\ ${ }^{1}$ Departamento de Física Aplicada, Universidade de Vigo, As Lagoas s/n, 32004 Ourense, Spain \\ ${ }^{2}$ Nonlinear Physics Center, Research School of Physics and Engineering, Australian National University, \\ Canberra ACT 0200, Australia
}

(Received 18 June 2010; accepted 4 August 2010; published online 24 August 2010)

A study on the nonlinear modes of plasmonic directional couplers created by two nonlinear dielectric slot waveguides with metallic claddings is presented. The calculated power diagram shows substantial differences from the case of nonlinear dielectric couplers. The power-dependent switching and the effect of losses on the coupler performance are studied using finite-difference time-domain numerical simulations. (C) 2010 American Institute of Physics.

[doi:10.1063/1.3482939]

The interest to the study of surface plasmon polaritons ${ }^{1}$ was renewed recently with a rapid development of nanofabrication in the hope that plasmonic circuits may revolutionize integrated optics, increasing functionalities of basic operating elements in information processing. ${ }^{2,3}$ It is believed that incorporating metals into optical elements would allow overcoming the fundamental diffraction limits by squeezing light into the subwavelength scale, also making the photonic circuits compatible with nowadays electronics. One of the simplest plasmonic waveguides is an interface between a metal and a dielectric that supports surface plasmon waves; however, direct excitation of plasmons at an interface is not efficient. Introducing multilayer systems, such as metaldielectric-metal waveguides, allows to increase substantially the propagation distance, since light propagation is tightly confined in a slot waveguide created by metal slabs, and it can even be used for efficient nanofocusing. ${ }^{4}$

One of the key elements of integrated photonic circuits, a directional coupler, is based on cross-talk coupling between two neighboring waveguides. Such directional couplers for plasmonic waves have been studied theoretically and even demonstrated experimentally for different geometries. ${ }^{5-7}$ Directional couplers have also been suggested for converting the modes of an optical dielectric waveguide into plasmonic modes propagating along a thin metal stripe. ${ }^{8}$

Nonlinear plasmonic structures were known for many years to provide additional possibilities for the mode control. $^{9-11}$ Recently, it was shown that nonlinear slot waveguides created by a nonlinear dielectric slab sandwiched between two metals support a variety of nonlinear subwavelength guided modes of different symmetries, ${ }^{12}$ including a type of asymmetric modes that might be important for nonlinear switching.

In this paper, a study on the nonlinear switching phenomena in plasmonic directional couplers is presented, analyzing the mode structure and switching characteristics of two coupled nonlinear plasmonic slot waveguides. More specifically, a directional coupler created by two neighboring slot waveguides with nonlinear dielectric cores surrounded by metal is considered and its modes numerically calculated. After obtaining the power diagram a crossover between lin-

${ }^{a)}$ Electronic mail: jrs@uvigo.es. ear and nonlinear switching of gap plasmonic modes is demonstrated.

The Maxwell's equations for the electric $\mathcal{E}(\mathbf{R}, t)$ and magnetic $\mathcal{H}(\mathbf{R}, t)$ fields, where $\mathbf{R} \equiv(X, Y, Z)$ is the position vector and $t$ the time, are written in the form,

$$
\nabla \times \mathcal{E}=-\mu_{0} \partial_{t} \mathcal{H}
$$

$$
\nabla \times \mathcal{H}=\partial_{t} \mathcal{D}
$$

where $\mu_{0}$ is the vacuum magnetic permeability and $\mathcal{D}$ $=\epsilon_{0} \in \mathcal{E}$ the electric displacement, being $\epsilon(\mathbf{R})$ the dielectric function describing the medium and $\epsilon_{0}$ the vacuum permittivity. A planar coupler formed by two dielectric cores with a nonlinear Kerr response and separated a distance $d$, bounded by metallic linear claddings (see Fig. 1) is considered. Taking harmonic fields of frequency $\omega$, the variables are made di-
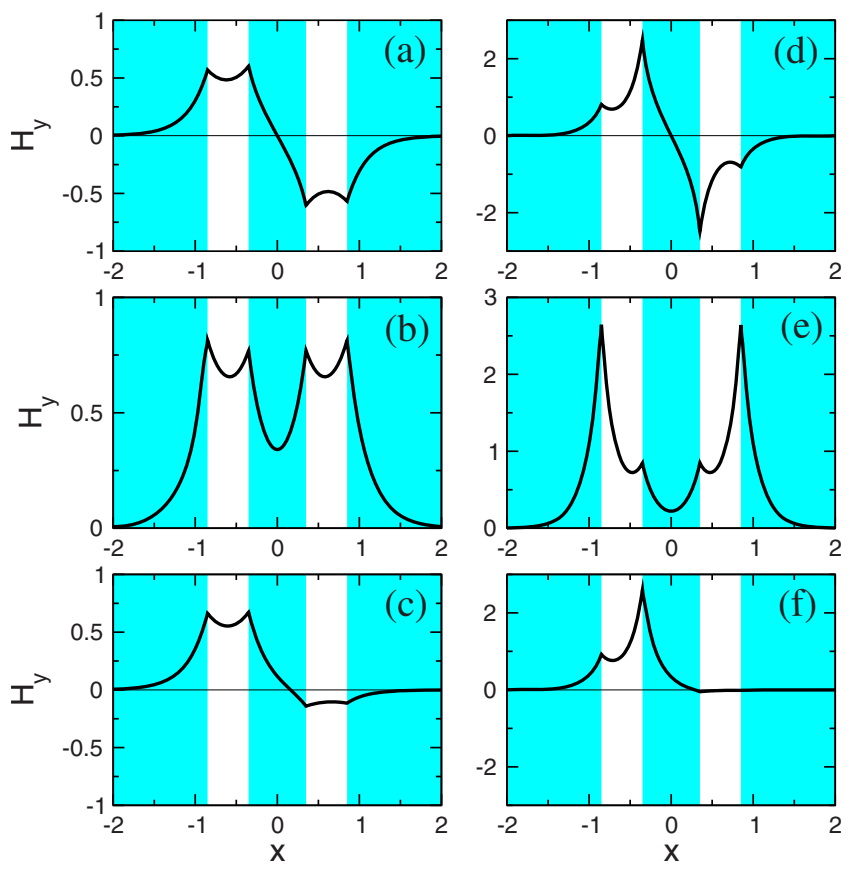

FIG. 1. (Color online) Examples of TM modes of a nonlinear slot coupler for $\beta=3.0$ (left column) and $\beta=5.0$ (right column). The plots show the magnetic component for antisymmetric modes [(a) and (d)], symmetric modes [(b) and (e)], and asymmetric modes [(c) and (f)]. The labels correspond to the points marked in Fig. 2. 
mensionless by scaling them as $\mathbf{r}=k_{0} \mathbf{R}$, where $\mathbf{r} \equiv(x, y, z)$ and $k_{0}=\omega / c$, being $c$ the speed of light in vacuum. The structure is assumed to lie in the plane $X Z$ and $z$ is taken as the propagation direction. Considering transverse magnetic (TM) polarization for the electromagnetic fields with components $\mathcal{H}_{y}, \mathcal{E}_{x}$, and $\mathcal{E}_{z}$ the layered medium is described using the following dielectric function:

$$
\epsilon(\mathbf{r})=\left\{\begin{array}{ll}
\epsilon_{m} ; & \text { for claddings } \\
\epsilon_{d}+\gamma\left(\left|\mathcal{E}_{x}\right|^{2}+\left|\mathcal{E}_{z}\right|^{2}\right) ; & \text { for cores }
\end{array},\right.
$$

where $\gamma$ accounts for the Kerr nonlinear response.

Stationary states are searched with the form,

$$
\begin{aligned}
& \mathcal{H}(\mathbf{r}, t)=\mathbf{H}(x) \exp [-i(\beta z-\omega t)] /\left(\mu_{0} c \sqrt{\gamma}\right), \\
& \mathcal{E}(\mathbf{r}, t)=\mathbf{E}(x) \exp [-i(\beta z-\omega t)] / \sqrt{\gamma},
\end{aligned}
$$

where $\beta$ is the propagation constant. The amplitudes of the fields have been normalized by two respective factors involving the system parameters in order to make them dimensionless magnitudes and further obtain the equations in a canonical form. Replacing Eqs. (4) and (5) in Eqs. (1) and (2) and considering TM-fields of the form $\mathbf{H}=H_{y} \hat{y}, \mathbf{E}=E_{x} \hat{x}$ $+i E_{z} \hat{z}$, the following $z$-independent system for the field components is obtained,

$$
\begin{aligned}
& \partial_{x} H_{y}=-\epsilon E_{z}, \\
& \partial_{x} E_{z}=\left(1-\beta^{2} / \epsilon\right) H_{y},
\end{aligned}
$$

together with the relationship $\epsilon E_{x}=\beta H_{y}$.

Inside the metallic regions, the dielectric function is negative $\left(\epsilon=\epsilon_{m}<0\right)$ and the equations above have an analytic solution for $H_{y}$ and $E_{z}$ in the form of a linear combination of terms containing $\exp \left( \pm \sqrt{\beta^{2}-\epsilon_{m}}\right)$ which is properly chosen to obtain a decaying behavior at $\pm \infty$. Inside the cores, using Eqs. (3)-(5), and the dependence $\epsilon E_{x}=\beta H_{y}$, the following cubic equation is obtained to express $\epsilon$ as a function of the field components $H_{y}$ and $E_{z}$,

$$
\epsilon^{3}-\left(\epsilon_{d}+E_{z}^{2}\right) \epsilon^{2}-\beta^{2} H_{y}^{2}=0 .
$$

It is easy to demonstrate that this cubic equation has only one real solution which can be obtained using Cardano's method. Once this solution is replaced in Eqs. (6) and (7) the system of ordinary differential equations is numerically solved by a relaxation method using as boundary conditions those that continuously connect the fields $H_{y}$ and $E_{z}$ at the boundaries between claddings and cores. Once the modes are numerically obtained at the nonlinear cores, they are analytically augmented to the metallic regions.

All the calculations are carried out taking $\epsilon_{d}=2.25, \epsilon_{m}$ $=-8.25$, (silver for a wavelength $\lambda \approx 480 \mathrm{~nm}$ ), ${ }^{13}$ waveguide width $w=0.5$ and separation between waveguides $d=0.7$. The modes are characterized by their power flux in the $z$-direction, $P=\int(\mathbf{E} \times \mathbf{H}) \hat{z} d x=\beta \int\left(H_{y}^{2} / \epsilon\right) d x$, and propagation constant. Examples of modes are shown in Fig. 1 for $\beta$ $=3.0$ and 5.0 and the power versus propagation constant diagram is shown in Fig. 2.

Three types of modes are found: symmetric [modes (a) and (d)], antisymmetric [modes (b) and (e)], and asymmetric [modes (c) and (f)]. In the linear limit, ${ }^{14}$ there exist both symmetric and antisymmetric modes [modes (a) and (b)] guided by the slot. They are created by in-phase or out-of-

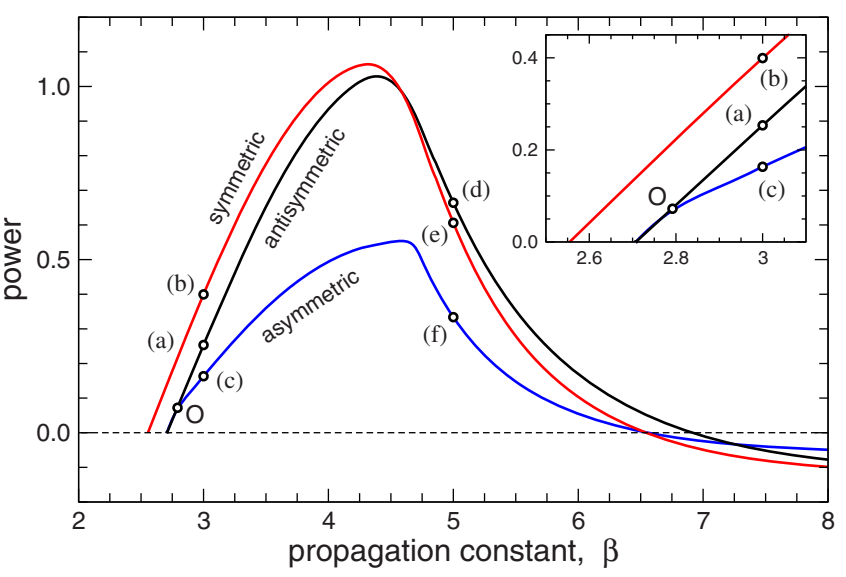

FIG. 2. (Color online) Total mode power vs propagation constant for the modes of the nonlinear plasmonic coupler. The points [(a)-(f)] correspond to the modes shown in Fig. 1. Point $\mathrm{O}$ is the bifurcation point for the asymmetric mode.

phase symmetric modes of isolated slot waveguides. Already for relative small power, a symmetry breaking takes place when the asymmetric mode [mode (c)] emerges at the bifurcation point $\mathrm{O}$ (see Fig. 2). This mode is still composed of two out-of-phase modes (since it bifurcates from a branch of antisymmetric solutions) but the field amplitude becomes different in the two slots.

For larger powers, all those three modes change almost independently, but the field becomes mainly confined at the boundaries between dielectric and metallic layers. Such a high field concentration leads to the symmetry breaking in each of the slot waveguides, described earlier for an isolated nonlinear slot, ${ }^{12}$ and the amplitudes at the metal-dielectric surfaces become different [modes (d) and (e)]. The field is shifted toward the metal cladding, so that the total power decreases, and it may even become negative due to the dominant (negative) contribution of the backward power flux concentrated in the metal (see Fig. 2), similarly to a single nonlinear slot waveguide. ${ }^{12}$

The dynamics of the nonlinear system is studied using finite-difference time-domain (FDTD) simulations. To account for the high dispersive metallic layers and make the algorithm numerically stable a classical model for cold plasmas is introduced in Eqs. (1) and (2) writing the displacement vector as $\mathcal{D}=\epsilon_{0} \mathcal{E}+\mathcal{P}$ and modeling the polarization current $\mathcal{J}=\partial_{t} \mathcal{P}$ by the equation, ${ }^{15}$

$$
\partial_{t} \mathcal{J}+\Gamma \mathcal{J}=\epsilon_{0} \omega_{p}^{2} \mathcal{E},
$$

where $\omega_{p}$ is the plasma frequency and $\Gamma$ is the electron collision frequency that accounts for the power losses. Both parameters can be calculated considering the Drude model $\epsilon(\omega)=1-\omega_{p}^{2} /\left(\omega^{2}+i \Gamma \omega\right)$ and using the values $\operatorname{Re}(\epsilon)=-8.25$ and $\operatorname{Im}(\epsilon)=0.3$, deduced from the optical constants of silver at $\lambda \approx 480 \mathrm{~nm}^{13}$ For $\omega=1$, i.e., measuring time in the units of $\omega^{-1}$, it is obtained $\omega_{p}=3.043$ and $\Gamma=0.032$. On the other hand, to model the dielectric cores, an instantaneous nonlinear response ${ }^{16}$ is considered using Eq. (3) together with Eqs. (1) and (2). With this approach numerical instabilities rise after a certain propagation time, which is shorter the larger the power is. Anyway, for a moderate power the instabilities are delayed a time quite long enough to perfectly observe the coupling and switching characteristics. These instabilities are due to the simple nonlinear model used and it is expected 

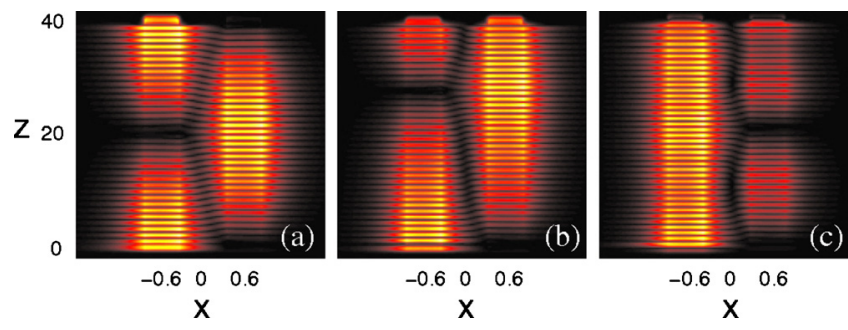

FIG. 3. (Color online) Examples of mode propagation and beating in the plasmonic coupler for different input powers: (a) Linear regime, $P_{0}=0.10$, (b) intermediate regime, $P_{0}=0.20$, and (c) nonlinear regime, $P_{0}=0.25$. The image shows the modulus of the magnetic component.

they are suppressed by using a more elaborated scheme.

The three images presented in Fig. 3 correspond to simulations carried out for different input power to illustrate how power flux can be switched from the second core to the first one just controlling input power. The FDTD simulations were performed by exciting one of the waveguides at $z=0$ with a hard source presenting the shape of the linear mode of the single-waveguide, properly scaled to the required power. After propagating for the necessary number of time-steps, further steps are calculated to time-average the Poynting vector, which is finally integrated in the transversal variable $x$ at $z=z_{b}$, where $z_{b}$ is the beat length, i.e., the distance at which power completely transfers to the second core under linear propagation. The switching curve $(\Gamma=0)$ is shown in Fig. 4,

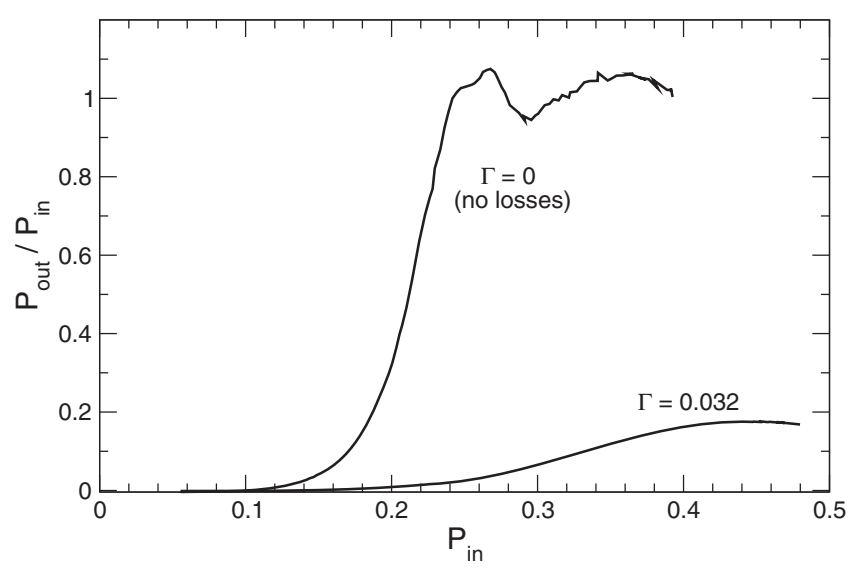

FIG. 4. Normalized output power $P / P_{0}$ vs input power $P_{0}$ for the excited waveguide of the nonlinear plasmonic slot coupler at the beat length $z_{b}$ $=19.26$, shown for vanishing and realistic losses. where the normalized output power $P / P_{0}$ is plotted against the input power $P_{0}$, showing how power is completely transferred from one core to the other when power raises over a threshold. Also, in this figure it is shown the switching curve when realistic losses are taken into account. As expected, there is a decrease in power in this case but switching is still possible. A particularly large distance between cores was used in order to achieve switching for a lower power avoiding the numerical instabilities. In practice, both slots in the coupler are closer to each other and consequently beat length is shorter and the effect of losses less important.

In conclusion, we have studied nonlinear modes and alloptical switching properties of planar directional couplers created by two weakly coupled slot metal-dielectric-metal waveguides. We have discussed nonlinear switching and demonstrated that the plasmonic coupler is still operative when realistic losses of metals are included.

This work was supported by the Australian Research Council and the Ministerio de Ciencia e Innovación of Spain through the Acción Complementaria Internacional under Grant No. PCI2006-A7-0561 and the Project No. MAT200806870 and the Ramón y Cajal contract granted to J.R.S.

${ }^{1}$ A. D. Boardman, Electromagnetic Surface Modes (Wiley, New York, 1982).

${ }^{2}$ S. A. Maier, Plasmonics: Fundamentals and Applications (Springer, New York, 2007).

${ }^{3}$ S. I. Bozhevolnyi, V. S. Volkov, E. Devaux, J. Y. Laluet, and T. W. Ebbesen, Nature (London) 440, 508 (2006).

${ }^{4}$ R. Yang, M. A. G. Abushagur, and Z. Lu, Opt. Express 16, 20142 (2008).

${ }^{5}$ D. K. Gramotnev, K. C. Vernon, and D. F. P. Pile, Appl. Phys. B: Lasers Opt. 93, 99 (2008).

${ }^{6}$ Z. Chen, T. Holmgaard, S. I. Bozhevolnyi, A. V. Krasavin, A. V. Zayats, L. Markey, and A. Dereux, Opt. Lett. 34, 310 (2009).

${ }^{7}$ T. Holmgaard, Z. Chen, S. I. Bozhevolnyi, L. Markey, and A. Dereux, J. Lightwave Technol. 27, 5521 (2009).

${ }^{8}$ A. Degiron, S. Y. Cho, T. Tyler, N. M. Jokerst, and D. R. Smith, New J. Phys. 11, 015002 (2009).

${ }^{9}$ V. M. Agranovich, V. S. Babichenko, and V. Y. Chernyak, Sov. Phys. JETP Lett. 32, 512 (1980).

${ }^{10}$ G. I. Stegeman, C. T. Seaton, J. Ariyasu, R. F. Wallis, and A. A. Maradudin, J. Appl. Phys. 58, 2453 (1985).

${ }^{11}$ D. Mihalache, G. I. Stegeman, C. T. Seaton, E. M. Wright, R. Zanoni, A. D. Boardman, and T. Twardowski, Opt. Lett. 12, 187 (1987).

${ }^{12}$ A. R. Davoyan, I. V. Shadrivov, and Y. S. Kivshar, Opt. Express 16, 21209 (2008).

${ }^{13}$ P. B. Johnson and R. W. Christy, Phys. Rev. B 6, 4370 (1972).

${ }^{14}$ M. Conforti, M. Guasoni, and C. De Angelis, Opt. Lett. 33, 2662 (2008)

${ }^{15}$ S. A. Cummer, IEEE Trans. Antennas Propag. 45, 392 (1997).

${ }^{16}$ R. M. Joseph and T. Allen, IEEE Photonics Technol. Lett. 6, 1251 (1994). 